\title{
A New Algorithm for Trademark Image Retrieval Based on Sub-block of Polar Coordinates
}

\author{
Bei-ji Zou ${ }^{1}$, Yi Yao ${ }^{1}$, and Ling Zhang ${ }^{2}$ \\ ${ }^{1}$ School of Information Science and Engineering, Central South University \\ Changsha, 410083, China \\ ${ }^{2}$ School of Computer and Communications, Hunan University \\ Changsha 410082, China
}

\begin{abstract}
The sub-block-based image retrieval method utilizes global and local image features to retrieve trademarks through separating individual an images into some blocks. This paper proposes a sub-block-based trademark image retrieval algorithm under the polar coordinate system. Experiment results show that our algorithm can keep excellent invariance in image translation, scaling and rotation and the retrieved results can satisfy human visual perception very well.
\end{abstract}

Keywords: trademark retrieval, sub-block image, mirror invariant performance.

\section{Introduction}

Trademark stands for reputation of enterprises and quality of products. For protecting the rights of the registered trademarks, we need to confirm the qualification of trademarks by checking the receptiveness and similarity between the trademarks that are being registered and those that have been registered. Due to the increase of the number of the registered trademarks, an accurate and effective automatic retrieving system needs to be established since the traditional method based on manual coding can hardly satisfy the real-time and accuracy requirement of retrieving.

Currently, there are many types of automatic trademark retrieving methods. Lam[1] used invariant moment and Fourier descriptor to describe the shape features of trademark images. The shape features such as comparative area, circularity and complexity were used to retrieve images by Eakins [2] and others. Kim [3] proposed an approach that retrieved image by using visual feature--the Zernike moment. Jau Ling Shih [4] described trademark images by using features such as deformation parameter, shape invariant moment and edge histogram, and he made the weighted computing to the general similarity. However, by these methods, only the overall features of images were considered but some special relations between individual images neglected.

Trademark image is firstly divided into several blocks through the way of quad-tree subdivision given by literature [5]. Then the image similarity is measured by extracting global features and features of every block. Compared to the approaches that extract only global features, the retrieval efficiency is enhanced by this method. 
However such method has to undergo rotation and it achieves invariance through matching the principal orientation of shapes. On the other hand experiment results show that after rotation and ratio alteration of images, certain discrepancy will occur to principal orientation of shapes, and the corresponding blocks of the related two images will not be identical in reality, which may seriously influence the retrieval effects. Furthermore, this method can't keep good image invariance. The problems above-mentioned are resolved perfectly by a new sub-block-based image retrieval algorithm under the polar coordinate system proposed by this paper through making the best use of the features of blocks. In addition, according to the fact that the edge patterns of trademarks are visually more perceptible than those in central parts, the power of the edge blocks are enlarged reasonably to improve the retrieval efficiency of the algorithm. Experiment results show that our algorithm can keep excellent invariance in image translation, rotation and scaling and the retrieved results match human visual perception very well.

\section{The Sub-block-Based Retrieval Algorithm Under The Polar Coordinate System}

The sub-block retrieval method under polar coordinate proposed in this paper will first normalize the trademark images, and set the minimum circumscribed circle of the object as the object region. Next, we will divide the image object into blocks under polar coordinate, calculate the shape histogram, and smooth it. Finally, the distances between images are computed by a new measurement of similarity so as to keep good image invariance.

\subsection{The Method for Computing the Shape Principal Orientation and Object Region}

Generally, differing from other images, the man made trademark image gives more importance to shape feature rather than color feature and texture feature. Therefore, in this paper only shape feature is utilized for data retrieval.

After pretreatment, a binary trademark image can be obtained. As shown in figure 1, the minimum circumscribed circle and shape principal orientation of object is computed, and the former is the object region of trademark image. Then a polar coordinate system is built up by considering the center of the minimum circumscribed circle as origin and shape principal orientation as polar pole. It can keep elementary invariance under translation and rotation processes. The steps are as follows:

1. Compute the minimum circumscribed circle

2. Compute the shape principal orientation $(\alpha)$

According to the angle ( $\beta$ ) between principal axis direction [7] and horizontal direction, it can be computed as below:

$$
\beta=\arctan \left(\left(\mu_{02}-\mu_{20}+\sqrt{\left(\mu_{02}-\mu_{20}\right)^{2}+4 \mu_{11}^{2}}\right) / 2 \mu_{11}\right) .
$$

$\mu_{p q}$ is the (p+q) step center moment of image. It is defined as 


$$
\mu_{p q}=\sum_{x} \sum_{y}(x-\bar{x})^{p}(y-\bar{y})^{q} f(x, y) .
$$

$(\bar{x}, \bar{y})$ is the center of mass of image. The $\alpha$ can be computed as follows:

$$
\alpha= \begin{cases}\beta+\pi, & u_{30}>0 \\ \beta, & u_{30} \leq 0\end{cases}
$$

\subsection{Division Method Under the Polar Coordinate System and Feature Extraction}

First the coordinate $(r, \theta)$ of pixels in object region should be computed, then let the coordinate of center of minimum circumscribed circle be $\left(x_{0}, y_{0}\right)$, and radius be $\mathrm{R}$

$$
\begin{gathered}
r=\sqrt{\left(x-x_{0}\right)^{2}+\left(y-y_{0}\right)^{2}}, \quad 0 \leqslant r \leqslant R . \\
\theta=\left(\arctan \left(\left(y-y_{0}\right) /\left(x-x_{0}\right)\right)-\alpha+2 \pi\right) \bmod (2 \pi), \quad 0 \leq \theta \leq 2 \pi .
\end{gathered}
$$

Figure 2 shows the blocking division of object region under polar coordinate. After dividing the object region into many blocks, the shape features of every block can be extracted. In this paper, the following two features are adopted to represent the shape of the divided block:

1) $e_{i}$ is denoted as the proportion of the object pixels in block which reflects the number of object pixels in No. i block, and the total pixels as $B_{i}$, then

$$
e_{i}=\sum_{(x, y) \in B_{i}} f(x, y) / \sum_{(x, y) \in B_{i}} 1
$$

2) $d_{i}$ is denoted as the distance between center of mass of block and origin, and $\left(x_{i 0}\right.$, $y_{i 0}$ ) as the center of mass of block $\mathrm{i}$, then

$$
d_{i}=\mid\left(x_{0}, y_{0}\right),\left(x_{i 0}, y_{i 0}\right) \mathrm{I} / R \text {. }
$$

The proportion histogram of pixels and distance histogram can be obtained by calculating the two shape features of every block and arranging these features according to their number. The histogram should be smoothed because the experiment results show that the shape principal orientations of two images have departure even though they are derived through rotating and scaling of the same image. The following is the smoothing method for the value of $i$ in histogram

$$
h_{i}=\sum_{j=i-k}^{j=i+k} h_{j} /(2 k+1)
$$

$h_{j}$ and $h_{i}$ are neighbors in the same cirque. 


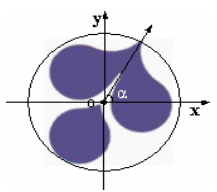

Fig. 1. Compute the minimum circumscribed circle and shape principal orientation

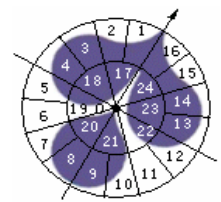

Fig. 2. Division method under the polar coordinate system

\subsection{Measurement of Comparability of Image}

Comparability among images can be measured according to global features and the individual block features. First, we discuss the measurement of individual block features (shape histogram).

The histograms distance between sample image A and image Q need to be checked

$$
D_{1}(A, Q)=\sum_{n} \sum_{i} \omega_{n}\left|h_{A, n, i}-h_{Q, n, i}\right|
$$

$\omega n$ is power of blocks on the nth cirque and hA,n,i is eigenvalue of image A in the ith block at the nth cirque. Besides, after mirror transformation, the order of each item in the histogram will be changed in relation to that of the original image. As shown in figure 3, the original anti-clockwise order is changed to clockwise one, and the distance between two histograms is

$$
D_{2}(A, Q)=\sum_{n} \sum_{i} \omega_{n}\left|h_{A, n, i}-h_{Q, n,(8 n+1-i)}\right|
$$

And the distance of shape histogram is as below:

$$
D(A, Q)=\min \left(D_{1}(A, Q), D_{2}(A, Q)\right)
$$

The values of invariant moments in Hu invariant moment group are different. To give the same treatment to these invariant moments, internal normalization is required and Gauss normalization is the method generally adopted.

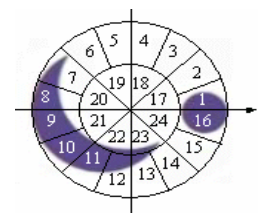

(a) The order of blocks of original image

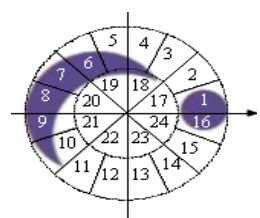

(b) The order of blocks of mirror image

Fig. 3. The corresponding relations among sub-blocks of mirror images 


$$
\phi_{i}{ }^{\prime}=\left\{\begin{array}{lc}
0, & \phi_{i}<\mu_{i}-3 \sigma_{i} \\
\frac{\phi_{i}-\mu_{i}+3 \sigma_{i}}{6 \sigma_{i}}, & \mu_{i}-3 \sigma_{i} \leq \phi_{i} \leq \mu_{i}+3 \sigma_{i} \\
1, & \phi_{i}>\mu_{i}+3 \sigma_{i}
\end{array}\right.
$$

Then the Euler distance of $\mathrm{Hu}$ invariant moment group and eccentricity distance of $\mathrm{A}$ and $\mathrm{Q}$ are computed. Now two shape histogram distances and two global feature distances are achieved. Before complex retrieval, the outer part of each distance should also be normalized. Next, compute the distance of every two images in database corresponding to one feature, then compute the excepted value and standard deviation of these distances, which should be normalized by Gauss normalization mentioned above. So the distance between sample image A and image Q that need to be checked is

$$
D(A, Q)=\sum_{i=1}^{4} \omega_{i} D_{i}, \quad \sum_{i=1}^{4} \omega_{i}=1
$$

$D_{i}$ is the distance of each feature after normalization. Experiment results show that retrieval outcome based on proportion histogram of pixels and distance histogram is more precise than that based on $\mathrm{Hu}$ invariant moment group and eccentricity. Therefore the power of two shape histograms should be a bit lager.

\section{Experimental Results}

To test the validity of algorithm in this paper, we performed several tests in trademark image database that contains 1000 binary trademark. The aim is to inspect the achieved geometry transform invariance of the algorithm proposed in this paper, and to see whether the retrieval effect satisfies visual requirement.

1) 50 trademark images belonging to different types from database are randomly chosen. Perform any combination of translation, rotation, scale, mirror and shape transformation to one of the selected image samples and we accordingly get four similar images to the original image. Then, we get a database including 250 trademark images that possesses 50 categories with each one containing five images. By taking any image from database as sample, the retrieval result returns the first 24 images arranged according to similarity.

As is shown in figure 4, it is clearly that other four images belonging to the same category with sample image are not arranged at the head of the range. And, the mirror image (the 12th and 14th in figure 4) is a bit far away from the first one (sample image).

Figure 5 shows the retrieval result of the same sample image by algorithm proposed in this paper. It shows that this algorithm can keep good geometry transformation invariance since images belonging to the same class with sample image are not only retrieved, but also arranged in the head of the range. 


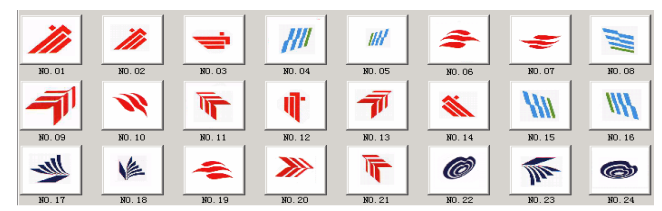

Fig. 4. The result of invariance experiment (based on quad-tree)

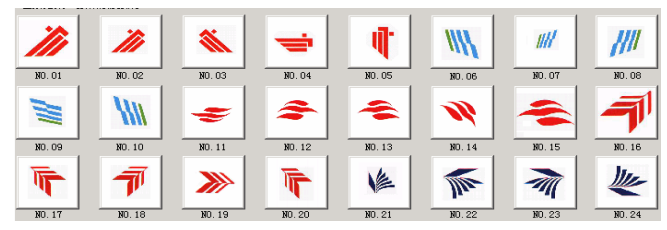

Fig. 5. The result of invariance experiment (under the polar coordinate)

3) Check the visual invariance of algorithm in this paper. In an image database that contains 1000 items, we choose anyone as sample and get the first 24 images arranged according to similarity. As is shown in figure 6 and 7, among images retrieved through quad-tree method (the 18th, 19th, 21st and 24th image), there are instances that differ from sample images obviously. But among the images achieved by algorithm in this paper, the construction and figure of most images are similar to the sample image. Furthermore, the more similar the images are, the nearer will they be arranged.

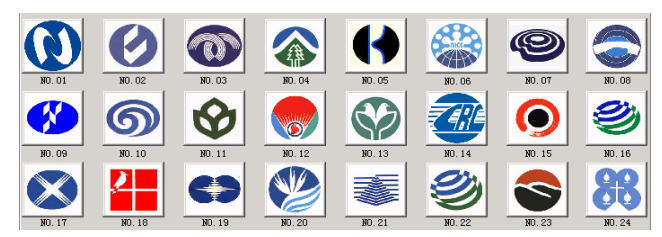

Fig. 6. The result of visual perception experiment (based on quad-tree)

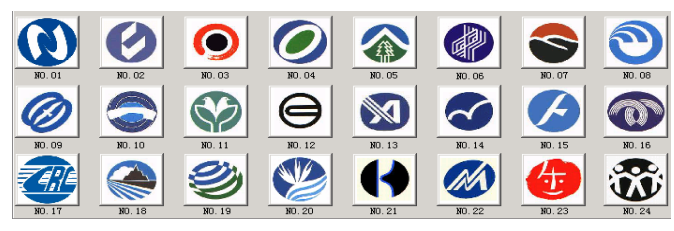

Fig. 7. The result of visual perception experiment (under the polar coordinate)

\section{Conclusion}

The sub-block based trademark image retrieval method utilizes both global and local features, which is certainly more efficient than most of the existing retrieval methods. 
Experiment results show that our method can keep good geometry transformation invariance and the human visual perception.

\section{Acknowledgement}

This research was supported by National Natural Science Fund (60673093).

\section{References}

1. Lam, C.P., Wu, J.K., Mehtre, B.: STAR - a system for Trademark archival and retrieval. In: Proceeding $2^{\text {nd }}$ Asian Conf. On Computer Vision, vol. 3, pp. 214-217 (1995)

2. Eakins, J.P., Boardman, J.M., Graham, M.E.: Similarity retrieval of trademark images. IEEE Transactions on Multimedia 5(2), 53-63 (1998)

3. Kim, Y.S., Kim, W.Y.: Content-based trademark retrieval system using a visually salient feature. Image and Vision Computing 16(12-13), 931-939 (1998)

4. Shih, J.L., Chen, L.H.: A new system for trademark segmentation and retrieval. Image and Vision Computing 19, 1011-1018 (2001)

5. Guo, L., Huang, Y.Y., Yang, J.Y.: Using sub-block image features to retrieve trademark image. Journal of Computer-Aided Design and Computer Graphics 16(17), 968-972 (2004)

6. Preparata, F.P., Shamos, M.L.: Computational Geometry: an Introduction. Springer, New York (1985)

7. Wang, T., Liu, W.Y., Sun, J.g., Zhang, H.J.: Using Fourier descriptors to recognize object's shape. Journal of Computer Research and Development 39(12), 1714-1719 (2002)

8. Wu, J.K.: Digital Image Analysis (in Chinese), pp. 28-31. People's Posts and Telecommunications Press, Beijing (1989) 